\title{
Influência da capacidade funcional e da presença de doenças crônicas não transmissíveis na autopercepção da saúde de idosas institucionalizadas
}

Influence of functional capacity and presence of chronic diseases not transmissible on the self-perception of the health of institutionalized elderly women

Influencia de la capacidad funcional y de la presencia de enfermedades crónicas no transmisibles en la autopercepción de la salud de ancianas institucionalizadas

Thayane Andressa Beltrão de Santana ${ }^{1}$, Wine Suélhi dos Santos ${ }^{1 *}$, Anna Júlia de Vasconcelos Moreira $^{2}$, Denise Rodrigues da Silva ${ }^{1}$, Bárbara Karolayne Mendonça dos Santos ${ }^{1}$, Nayara Carolynna Beltrão de Santana ${ }^{3}$, Pedro de Sousa Leite ${ }^{4}$, Lucas Tavares Cruz de Albuquerque ${ }^{4}$, Caroline Pereira Modesto ${ }^{4}$, Joseja Jaqueline de Medeiros $^{5}$, Pedro Ykaro Fialho Silva ${ }^{6}$, Raiana Fernanda da Silva Santos ${ }^{7}$, Cristália Emília Bias de Oliveira ${ }^{8}$.

\section{RESUMO}

Objetivo: Verificar a influência da capacidade funcional e da presença de doenças crônicas não transmissíveis (DCNT) na autopercepção da saúde de idosas de uma instituição de longa permanência para idosos (ILPI) na cidade do Recife/PE, Brasil. Métodos: Trata-se de um estudo descritivo, transversal e de caráter quantitativo, composto por 22 idosas. A pesquisa foi dividida em três etapas: I- Entrevista semiestruturada com a utilização das sessões I, II, V e VI do questionário Brazil Old Age Shedule (BOAS); II- Avaliação da capacidade funcional pela Medida de Independência Funcional (MIF); III- Análise dos prontuários médicos para verificação das DCNT. A estatística foi realizada por meio do software IBM SPSS versão 20.0 adotando-se significância estatística de $p<0,05$. Estudo aprovado sob CAAE 67687317.1.0000.5640. Resultados: $O$ teste de Spearman mostrou correlação de $r=-0,733 ; p=0,0001$ entre os escores da MIF e a autopercepção de saúde categorizado em "Boa" e "Ruim", ou seja, a saúde avaliada como ruim está associada com menores pontuações na MIF (Teste de Mann-Whitney $\mathrm{p}=0,001$ ). Nas DCNT e autopercepção de saúde, o teste de Spearman mostrou correlação de $r=0,670 ; p=0,001$, o que significa que a autopercepção ruim de saúde avaliada pelas idosas está associada com uma maior incidência de DCNT (Teste de Mann-Whitney $p=0,001$ ). Conclusão: As idosas que possuem doenças crônicas e perda funcional tendem a autoperceber a saúde negativamente.

Palavras-chave: Saúde do Idoso, Autopercepção, Funcionalidade, Doenças Crônicas não Transmissíveis.

\footnotetext{
${ }^{1}$ Fisioterapeutas residentes em Terapia Intensiva no Hospital Dom Hélder Câmara (HDH), Cabo de Santo Agostinho-PE. *E-mail: winesuelhi@gmail.com

${ }^{2}$ Fisioterapeuta pós graduanda em Terapia Intensiva no Centro de Formação e Aperfeiçoamento Profissional de Recife (CEFAPP), Recife-PE.

${ }^{3}$ Fisioterapeuta graduada pela Faculdade Maurício de Nassau de Recife (UNINASSAU), Recife-PE.

${ }^{4}$ Discentes do curso de Medicina da Faculdade de Medicina Estácio de Juazeiro do Norte (FMJ), Juazeiro do Norte-CE.

${ }^{5}$ Fisioterapeuta residente em Saúde Coletiva na Universidade Regional do Cariri (URCA), Crato-CE.

${ }^{6}$ Fisioterapeuta graduado pelo Centro Universitário Dr. Leão Sampaio (UNILEÃO), Juazeiro do Norte-CE.

${ }^{7}$ Enfermeira no Hospital Dom Hélder Câmara (HDH) e Mestranda em Engenharia Biomédica pela Universidade Federal do Pernambuco (UFPE), Cabo de Santo Agostinho-PE.

${ }^{8}$ Fisioterapeuta docente da Faculdade Maurício de Nassau de Recife (UNINASSAU), Recife-PE.
} 


\begin{abstract}
Objective: To verify the influence of functional capacity and the presence of chronic diseases not transmissible (CDNT) on the self-perception of the health of elderly women in a long-term institution for the elderly (ILPI) in the city of Recife/PE, Brazil. Methods: This is a descriptive, cross-sectional, quantitative study of 22 elderly women. The research was divided into three stages: I- Semi-structured interview using sessions I, II, V and VI of the Brazil Old Age Shedule questionnaire (BOAS); II- Evaluation of functional capacity by Functional Independence Measure (MIF); III- Analysis of medical records for verification of CDNT. Statistical analysis was performed using the IBM SPSS software version 20.0, adopting a statistical significance of $p<0.05$. Study approved under CAAE 67687317.1.0000.5640. Results: The Spearman test showed a correlation of $r=-0.733 ; p=0.0001$ between MIF scores and self-rated health categorized as "Good" and "Bad", that is, poor health is associated with lower MIF scores (Mann-Whitney test $p=0.001$ ). In the CDNT and self-perception of health, the Spearman test showed a correlation of $r=0.670 ; p=0.001$, which means that poor self-rated health evaluation by elderly women is associated with a higher incidence of CDNT (Mann-Whitney test $p=0.001$ ). Conclusion: The elderly with chronic diseases and functional loss tend to perceive health negatively.
\end{abstract}

Keywords: Elderly Health, Self-perception, Functionality, Chronic Diseases not Transmissible.

\title{
RESUMEN
}

Objetivo: Verificar la influencia de la capacidad funcional y de la presencia de enfermedades crónicas no transmisibles (DCNT) en la autopercepción de la salud de ancianos de una institución de larga permanencia para ancianos (ILPI) en la ciudad de Recife/PE, Brasil. Métodos: Se trata de un estudio descriptivo, transversal y de carácter cuantitativo, compuesto por 22 ancianas. La investigación se dividió en tres etapas: I- Entrevista semiestructurada con la utilización de las sesiones I, II, V y VI del cuestionario Brazil Old Age Shedule (BOAS); II - Evaluación de la capacidad funcional por la Medida de Independencia Funcional (MIF); III - Análisis de los prontuarios médicos para verificación de las DCNT. La estadística fue realizada a través del software IBM SPSS versión 20.0 adoptando significancia estadística de $p<0,05$. Estudio aprobado CAAE 67687317.1.0000.5640. Resultados: La prueba de Spearman mostró correlación de $r=-0,733 ; p=0,0001$ entre los escores de la MIF y la autopercepción de salud categorizada en "Buena" y "Mala", es decir, la salud evaluada como mala está asociada con menores puntuaciones en la MIF (Prueba de Mann-Whitney $\mathrm{p}=0,001$ ). En las $\mathrm{DCNT}$ y autopercepción de salud, la prueba de Spearman mostró correlación de $r=0,670 ;=0,001$, lo que significa que la autopercepción mala de salud evaluada por las personas mayores está asociada con una mayor incidencia de DCNT (Prueba de Mann-Whitney $\mathrm{p}=0,001$ ). Conclusión: Las ancianas que poseen dolencias crónicas y pérdida funcional tienden a autoperceptar la salud negativamente.

Palabras claves: Salud del Anciano, Autopercepción, Funcionalidad, Enfermedades Crónicas no Transmisibles.

\section{INTRODUÇÃO}

Envelhecer é um processo natural e inerente ao ser humano, estando o contingente populacional de idosos no Brasil crescendo acentuadamente desde o século $X X$, fator que pode ser atribuido as baixas taxas de fecundidade dos últimos anos associado com o aumento da expectativa de vida e redução das taxas de mortalidade (ALVES LC e RODRIGUES RN, 2005; DIAS DSG, et al., 2013). Projeções indicam o Brasil como sexto país no mundo em número de idosos para o ano de 2020 (VERAS R, 2009). Dados do Instituto Brasileiro de Geografia e Estatística apontam que sua população com 60 anos ou mais seja de, aproximadamnete, 73 milhões no ano de 2060 (IBGE, 2013).

O envelhecimento é uma questão de saúde pública, pois gera declínio das funções do organismo, por meio da atuação direta/indireta dos fatores genéticos e ambientais, além de outras características que 
individualizam o processo de envelhecer de cada organismo. Desta forma muitos são levados para Instituições de Longa Permanência para Idosos (ILPI) para receberem cuidados específicos ou, simplesmente, porque não existe outrem, como familiares, que possam prestar assistência a estes (BORGES AM, et al., 2014).

As ILPI são instituições governamentais ou não governamentais, de caráter residencial, destinadas ao domicílio coletivo de pessoas com idade igual ou superior a 60 anos, com ou sem suporte familiar, em condição de liberdade, dignidade e cidadania (ANVISA, 2005). Entre as causas de institucionalização de idosos estão solidão, necessidades de cuidados com a saúde, conflitos familiares e perdas de entes queridos (BESSA MEP e SILVA MJ, 2008).

Estudos apontam uma alta incidência de doenças crônicas não transmissíveis (DCNT), utilização de medicamentos, dependência física para locomoção, deficiências físicas e cognitivas nos idosos, como fatores que podem correlacionar-se com a institucionalização em ILPI (LIMA CLJ, et al., 2013). No Brasil as ILPI estão em sua maioria relacionadas com a dependência de idosos com necessidade de assistência, tornando assim importante avaliar seu estado de saúde com uma abordagem multidimensional que englobe os diversos aspectos de suas vidas (DANTAS CMHL, et al., 2013).

Indicadores de saúde simples para avaliar as circunstâncias de saúde da população idosa tem sido cada vez mais instituidos, nos países desenvolvidos e em desenvolvimento, para acompanhar o rápido processo de envelhecimento populacional (LIMA-COSTA MF, et al., 2004). Porquanto, a autopercepção de saúde vem sendo frequentemente utilizada na abordagem da associação entre saúde, determinantes demográficos e socioeconômicos, doenças crônicas e capacidade funcional em idosos, considerada um método capaz de expressar vários aspectos da saúde física, cognitiva e emocional dos indivíduos. Além disso, outros estudos indicam a autopercepçao de saúde como um meio crescente de utilização nas pesquisas de saúde geral e predição de saúde (ARNADOTTIR SA, et al., 2011).

A autoavaliação ou autopercepção de saúde é um importante indicador de morbidade, declínio funcional e mortalidade, abrangendo as dimensões social, psicológica e biológica do indivíduo avaliado (ALVES LC e RODRIGUES RN, 2005; BORGES AM, et al., 2014; DAMIÁN J, et al., 2008), pesquisas indicam forte relação entre a capacidade funcional e doenças crônicas com a autopercepção de saúde sendo considerados como alguns dos principais determinantes deste indicador (NUNES APN, et al., 2012; BELÉM PLO, et al., 2016).

Perante uma grande e crescente população idosa, fica evidente a necessidade da utilização de indicadores de saúde que norteiem intervenções e apontem as principais necessidades destes indivíduos, bem como compreender suas principais demandas e parâmetros para planejamento de estratégias que visem a melhor abordagem e assistência à saúde desta população. Diante do exposto o presente estudo teve como objetivo principal verificar a influência da capacidade funcional e da presença de DCNT na autopercepção da saúde de idosas institucionalizadas em uma ILPI na cidade do Recife/PE, Brasil.

\section{MÉTODOS}

Trata-se de um estudo descritivo, transversal e de caráter quantitativo, desenvolvido no Instituto Padre Venâncio, uma instituição de cunho privado e filantrópico, localizado na cidade do Recife, PernambucoBrasil, onde residem 60 mulheres. A coleta de dados foi realizada durante o período de maio a junho de 2017.

Os critérios de elegibilidade utilizados foram idosas com 60 anos ou mais, residentes na ILPI citada, que aceitassem participar da pesquisa e apresentassem um bom nível cognitivo avaliado pelo Mini-Exame do Estado Mental (MEEM).

Em relação ao teste do MEEM foram adotados os escores estabelecidos por Bertolucci PHF, et al. (1994) que consideram pontuação igual ou superior a 13 pontos para analfabetos, 18 para baixa ou média escolaridade e 26 para nível superior. 
Dentre as 60 idosas residentes na ILPI, duas vieram a óbito antes do início da pesquisa, uma mudou-se para residência de familiares, 13 se recusaram a participar da pesquisa e 22 não atingiram a pontuação mínima no MEEM e/ou possuíam comprometimento cognitivo grave devido à presença de doenças psicológicas e/ou neurológicas, não sendo, portanto, incluídas na amostra, fazendo com que o $\mathrm{n}$ amostral fosse constituído por 22 idosas.

A pesquisa foi dividida em três etapas: I- Entrevista semiestruturada com a utilização das sessões I, II, V e VI do questionário Brazil Old Age Shedule (BOAS) que originalmente é composto por nove sessões em um total de 113 questões para verificar as correlações da autopercepção de saúde com determinantes demográficos e socioeconômicos; II- Avaliação da capacidade funcional por meio do teste de Medida de Independência Funcional (MIF) que contém 18 itens que se agrupam em seis dimensões: autocuidado, locomoção, transferência, comunicação, controle dos esfíncteres e cognição social; III- Análise dos prontuários médicos das partícipes para verificação das DCNT diagnosticadas.

No questionário BOAS as variáveis independentes foram listadas da seguinte forma: I-Informações gerais, consideradas como determinantes demográficos (idade, escolaridade, estado conjugal, número de filhos, satisfação com a vida); II- Saúde física (a variável dependente: autopercepção de saúde, e as demais variáveis independentes: comparação da saúde com os útimos cinco anos, comparação da saúde com outras pessoas da mesma idade, presença de quedas nos últimos três meses, estado da visão, estado da audição, estado dos dentes e episódios de perda de urina); V- Recursos sociais (relacionamento com as pessoas que moram junto, assistência familiar e visitas na última semana) e VI- Recursos econômicos (ocupação anterior, trabalho atual, forma de sustento, renda mensal, comparação com situação econômica de 50 anos, avaliação de seu rendimento). A variável dependente foi a autopercepção de saúde, dicotomizada em boa (quando a resposta foi muito boa ou boa) e ruim (quando a resposta foi regular, ruim ou muito ruim) (WEISS SLI, 2013).

A capacidade funcional avaliada pelos pesquisadores com o uso da MIF pode variar de 18 a 126 pontos, sendo classificada com o escore de: 18 - dependência completa; 19 a 60 - dependência modificada (assistência de até 50\% das tarefas); 61 a 103 - dependência modificada (assistência de até 25\% das tarefas) e 104 a 126 - independência completa/modificada (BORGES JBC, 2006).

Já a presença de doenças crônicas foram embasadas nas DCNT citadas no levantamento feito pela Pesquisa Nacional da Saúde de 2013 que engloba: hipertensão arterial sistêmica (HAS), doenças cardiovasculares (DCV), Acidente Vascular Cerebral (AVC), diabetes, asma (sendo adicionado: ou outras doenças pulmonares crônicas), problema crônico de coluna, colesterol alto, Distúrbios Osteomusculares Relacionados ao Trabalho (DORT), depressão, insuficiência renal crônica e câncer, foi ainda adicionada uma opção "outros" para direcionar os problemas de saúde crônicos não listados (IBGE, 2014).

A análise estatística foi realizada por meio do software IBM SPSS (Statistical Package for Social Sciences) versão 20.0 para MacOs. Para determinar se a distribuição das variáveis era normal ou não utilizou-se o teste de Shapiro Wilk, evidenciando dados não paramétricos, desta maneira, foi aplicado o coeficiente de correlação de postos de Speraman e o teste de Mann-Whitney, entretanto, para os determinantes demográficos, de saúde, sociais e econômicos em comparação com a autopercepção de saúde utilizou-se teste qui-quadrado, adotando para os testes significância estatística um $p<0,05$, com um intervalo de $95 \%$ de confiança, além do uso da estatística descritiva com frequências relativas e percentis.

Para que os aspectos da ética e legalidade da pesquisa com humanos sejam rigorosamente cumpridos o estudo segue os preceitos éticos descritos conforme a Resolução 466/2012 que dispões sobre as diretrizes e normas regulamentadoras de pesquisas envolvendo seres humanos, normatizada pelo Conselho Nacional de Saúde - CNS/MS (BRASIL, 2012). Todas as informações do estudo foram fornecidas às participantes e logo após o aceite assinaram o Termo de Consentimento Livre e Esclarecido (TCLE). A pesquisa foi aprovada pelo Comitê de Ética em Pesquisa com Seres Humanos da Faculdade Estácio do Recife sob o CAAE 67687317.1.0000.5640 e parecer número 2066669. 


\section{RESULTADOS E DISCUSSÃO}

A amostra foi composta por 22 idosas com média de idade de $79,95 \pm 7,31$ anos, dentre as quais: 08 (36,36\%) avaliaram a saúde como boa, (03 "muito boa" e 05 "boa") e 14 (63,64\%) avaliaram a saúde como ruim (10 "regular", 03 "ruim" e 01 "muito ruim"). As idosas com a saúde autopercebida como boa

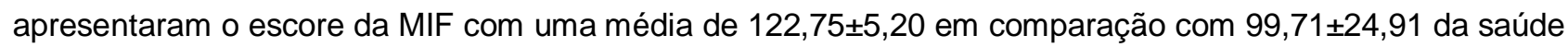
autopercebida como ruim (Tabela 1).

Tabela 1 - Autopercepção de saúde e Medida de Independência Funcional.

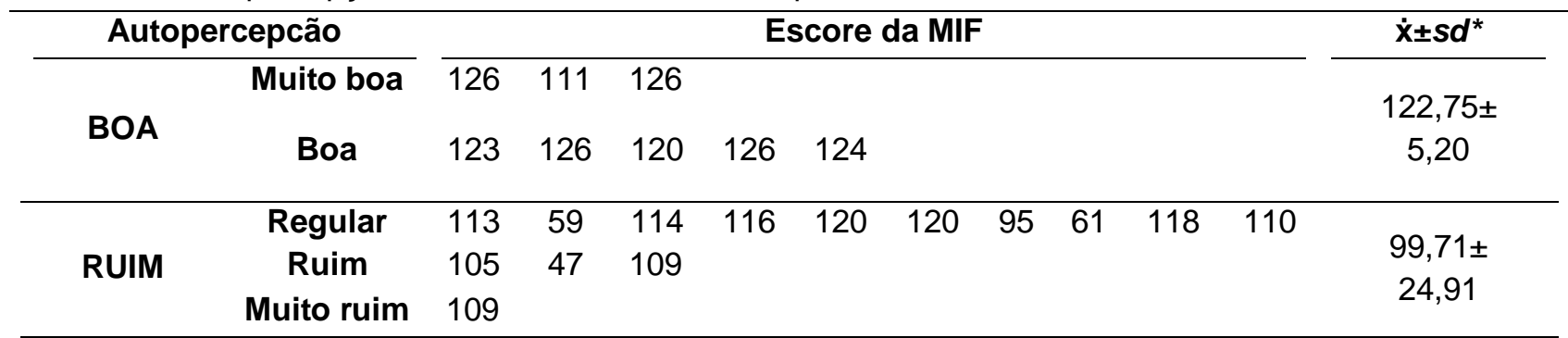

*Nota: $\dot{x} \pm s d$ (média \pm desvio padrão da média).

Fonte: Dados dos Autores, 2018.

O teste de correlação de Spearman mostrou correlação de $r=-0,733 ; p=0,0001$ entre os escores da MIF e a autopercepção de saúde categorizado em "Boa" e "Ruim", ou seja, a saúde avaliada como ruim pelas idosas está associada com menores pontuações na MIF. Na comparação das medianas através do teste de Mann-Whitney a funcionalidade dos indivíduos que tiveram autopercepção de saúde "Boa" e "Ruim" demonstrou $\mathrm{p}=0,001$ (Figura 1).

Este achado corrobora com o estudo de Alves LC e Rodrigues RN (2005) verificando-se que quanto maior o grau de dependência que o idoso apresenta, maior é a probabilidade dele autoperceber a sua saúde negativamente, embora este estudo tenha verificado também que quando comparado aos homens, as mulheres idosas tinham menores chances de avaliar negativamente a saúde.

Belém PLO, et al. (2016) também verificaram a mesma correlação, demonstrando que as incapacidades e dificuldades impostas por limitações funcionais impactam negativamente na avaliação da saúde dos idosos.

No estudo realizado por Damián J, et al. (2008), em lares públicos e privados para idosos na cidade de Madrid, na Espanha, os indivíduos analisados com qualquer grau de dependência, quando comparados aos funcionalmente independentes, tinham entre 1,81 e 2,37 vezes maiores chances de pior percepção de sua saúde.

Nunes APN, et al. (2012) observaram que tanto os idosos que tinham pouca dificuldade, quanto os que tinham grande dificuldade e não conseguiam realizar as atividades de vida diária (AVD); assim como os que avaliaram que dependiam muito e dependiam totalmente de outras pessoas, entre outros fatores, demonstravam maiores probabilidades de declarar sua saúde como ruim.

Alves LC, et al. (2010) ao pesquisarem sobre os fatores associados à incapacidade funcional de idosos no Brasil verificou que a autopercepção de saúde estava fortemente relacionada à incapacidade funcional, apresentando chances de maiores graus de dependência em idosos que fizeram uma avaliação ruim da sua saúde.

Belém PLO, et al. (2016) explicam que a autopercepção negativa de saúde para as mulheres pode estar mais associada com qualidade de vida, devido ao fato destas viverem mais que os homens e conviverem mais anos com doenças e incapacidades, tendendo-as a uma avaliação negativa. 
Figura 1 - Autopercepção de saúde e correlação da medida de independência funcional.

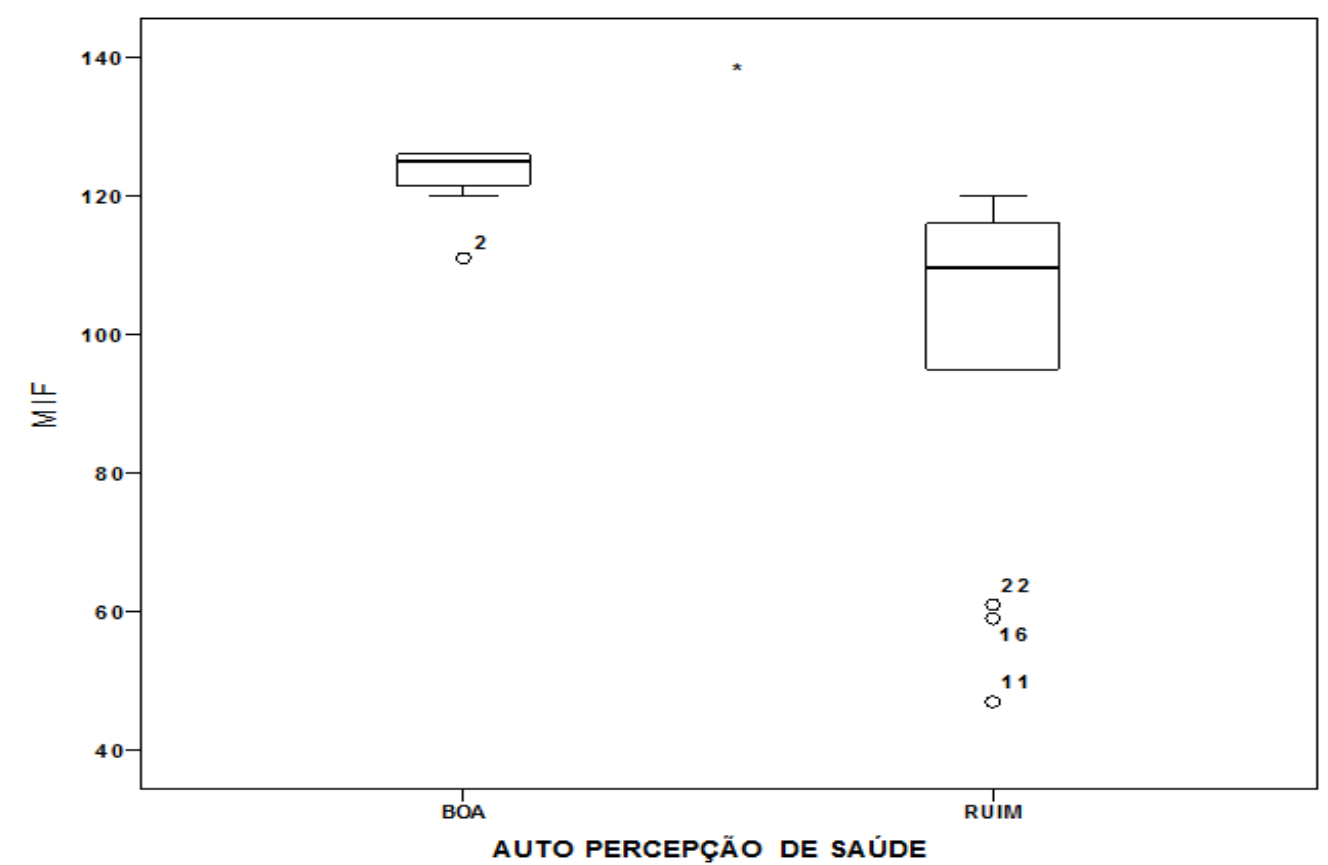

Fonte: Dados dos Autores, 2018.

Na relação entre DCNT e autopercepção de saúde, o teste de Spearman mostrou correlação de $r=0,670$; p=0,001 entre o número de doenças crônicas e a autopercepção de saúde categorizado em "Boa" e "Ruim", o que significa que a autopercepção ruim de saúde avaliada pelas idosas está associada com uma maior incidência de DCNT. Na comparação das medianas através do teste de Mann-Whitney a funcionalidade dos indivíduos que tiveram autopercepção de saúde "Boa" e "Ruim" demonstrou p=0,001 (Figura 2).

Dentre as DCNT mais prevalentes estão: 16 (72,7\%) de HAS, 11 (50\%) de diabetes, e 9 (40,9\%) com problemas crônicos de coluna. O grupo que autopercebeu sua saúde como boa apresentou uma média de

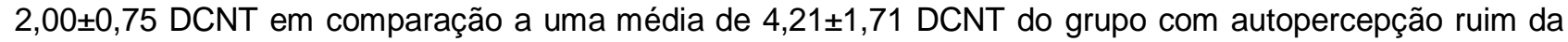
saúde. Damián J, et al. (2008) observaram que a cada condição crônica extra que era relatada, as chances de perceber pior a sua saúde aumentavam $18 \%$.

No Brasil entre as variáveis avaliadas como determinantes da autopercepção de saúde entre idosos, no estudo de Alves LC e Rodrigues RN (2005), encontravam-se as doenças crônicas e foi verificado que idosos nas categorias "uma doença crônica" e "quatro ou mais doenças crônicas" apresentaram um risco 1,86 e 11,98 vezes maior, respectivamente, de autoperceber a sua saúde como ruim em relação àqueles que não apresentavam doenças. O mesmo estudo mostrou ainda que a saúde autopercebida como ruim foi maior entre os idosos que apresentavam quatro ou mais doenças crônicas e que isso ocorreu tanto para os homens ( 9,5 vezes) quanto para as mulheres (7,3 vezes).

Em concordância Nunes APN, et al. (2012) relatam que entre fatores relevantes associados a uma maior probabilidade de autopercepção de saúde como ruim estão aqueles idosos que informaram ter duas ou mais doenças crônicas.

Em discordância destes que foram citados, o estudo de Borges AM, et al. (2014) destaca que apesar da maioria dos idosos ter referido boa saúde, grande número deles relatou ser portador de doenças crônicas, o que reforça a ideia de que a percepção de ser doente está mais relacionada às incapacidades do que a ser portador de danos crônicos. O referido autor atenta também ao fato de que muitas vezes, por ainda não possuírem sintomas, incapacidades ou comorbidades decorrentes das doenças crônica, acabam não se julgando doentes. 
Gráfico 2 - Autopercepção da saúde e coleção de Doenças Crônicas não Transmissíveis.

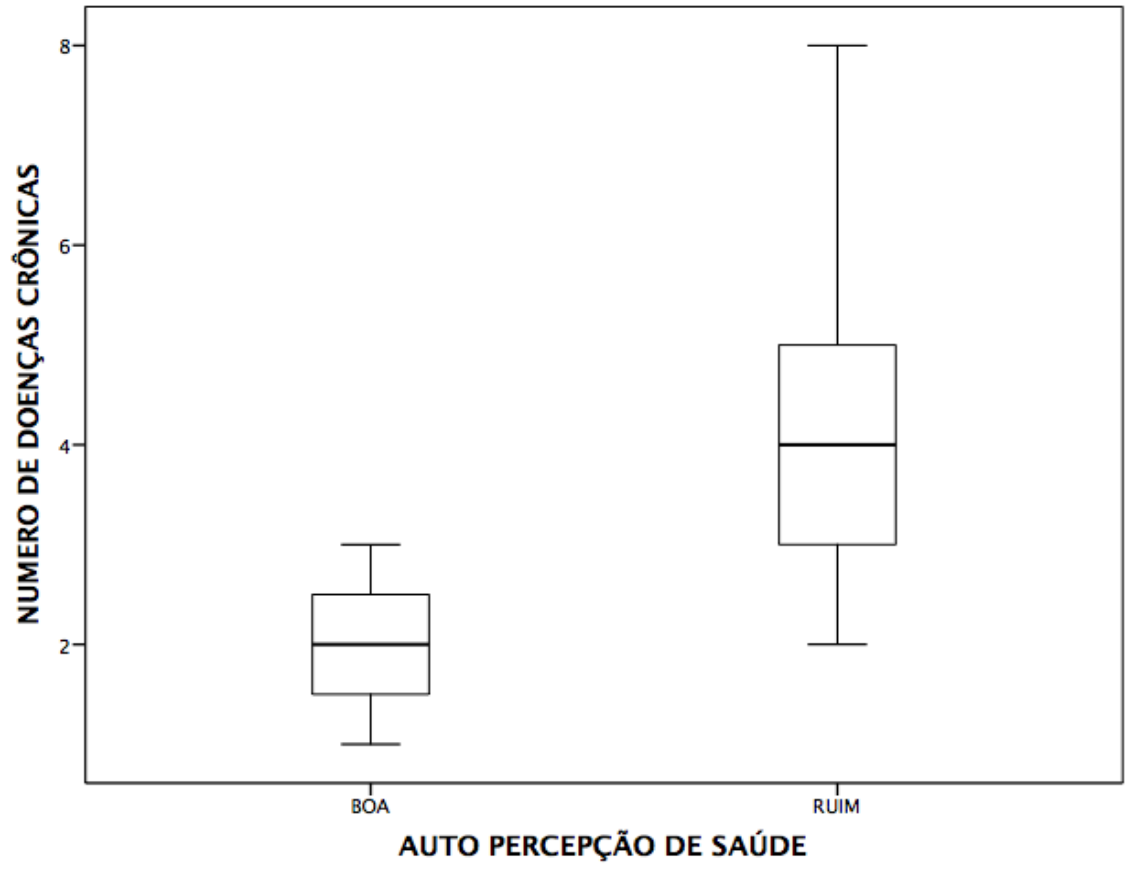

Fonte: Dados dos Autores, 2018.

Nos resultados dos demais determinates: demográficos, de saúde, sociais e econômicos a única variável que apresentou um resultado estatisticamente significante $(\mathrm{p}<0,05)$ foi a de "visitas na última semana" ( $p=0,039)$, que representa incidência de $92,9 \%$ de idosas que receberam visitas e que avaliaram a saúde como ruim (Tabela 2 ).

A amostra apresentou uma maioria (14 idosas) que autopercebeu a saúde como ruim, dentre elas, a maioria possui 80 anos ou mais (50\%), estudou até o primário (35,7\%), é viúva (57,1\%), número de filhos com mediana 0,5 e IQ (0-2), está satisfeita com a vida (64,3\%), julgam pior sua saúde comparada aos últimos 5 anos (64,3\%), julgam sua saúde igual (42,9\%) ou melhor (42,9\%) em comparação as pessoas da mesma idade, ausência de quedas nos últimos 3 meses $(71,4 \%)$, estado da visão ruim (50\%), estado da audição como boa (35,7\%) e ótima (35,7\%), estado dos dentes ruins (50\%), ter episódios de perda de urina (64,3\%). Em relação às variáveis socioeconômicas a maioria está satisfeita no relacionamento com as pessoas que mora $(92,9 \%)$, possui assistência familiar $(57,1 \%)$, receberam visitas na última semana (92,9\%), trabalhava anteriormente $(85,7 \%)$, mas nenhuma possui trabalho atual (100\%), como forma de sustento possuem a aposentadoria $35,7 \%$ e aposentadoria com a ajuda de familiares $35,7 \%$, possuem renda mensal de um salário mínimo ou menos (50\%), julgam a situação econômica atual igual a de quando tinham 50 anos $(57,1 \%)$ e por fim, que sua renda mensal em relação as suas necessidades básicas supre a demanda (50\%) (Tabela 2).

Alves LC e Rodrigues RN (2005) verificaram que a avaliação mais positiva de saúde é feita pelos idosos que moram sozinhos comparado aos que moram acompanhados, o que reforça que o sentimento de independência influencia positivamente na autopercepção de saúde. Entretanto, Nunes APN, et al. (2012) mostraram que a autopercepção da saúde ruim possuía uma associação positiva com relatos como ficar muito tempo sozinho, de insatisfação em seus relacionamentos pessoais e a sentimentos de falta de amparo e apoio.

O fato de quase todas as outras variáveis estudadas não se mostrarem estatisticamente significantes aponta que, embora outros estudos tenham constatado a estrutura multidimensional da autoavaliação de saúde (LIMA-COSTA MF, et al., 2004) e atestado a influência de determinantes socioeconômicos e de saúde na avaliação da saúde dos idosos como nível de escolariadade e renda (ALVES LC e RODRIGUES 
RN, 2005), problemas nos relacionamentos sociais (NUNES APN, et al., 2012) e idade (BELÉM PLO, et al., 2016), por exemplo, foi observado no presente estudo que são os fatores relacionados à presença de DCNT e limitações funcionais, bem como o impacto destas na vida dos idosos que geram maior peso e significância na autoavaliação. Isso também pode ser demostrado observando que mesmo as idosas recebendo visitas na última semana, relataram a sua saúde como ruim, lembrando que a este mesmo grupo está associado a uma convivência com um maior número de DCNT e menores escores funcionais, o que reforça a idéia de que são estas últimas que influenciam de forma mais significativa na hora da idosa avaliar sua saúde.

Tabela 2 - Determinantes demográficos, de saúde, sociais e econômicos em comparação com a autopercepção de saúde (Teste de Qui-Quadrado).

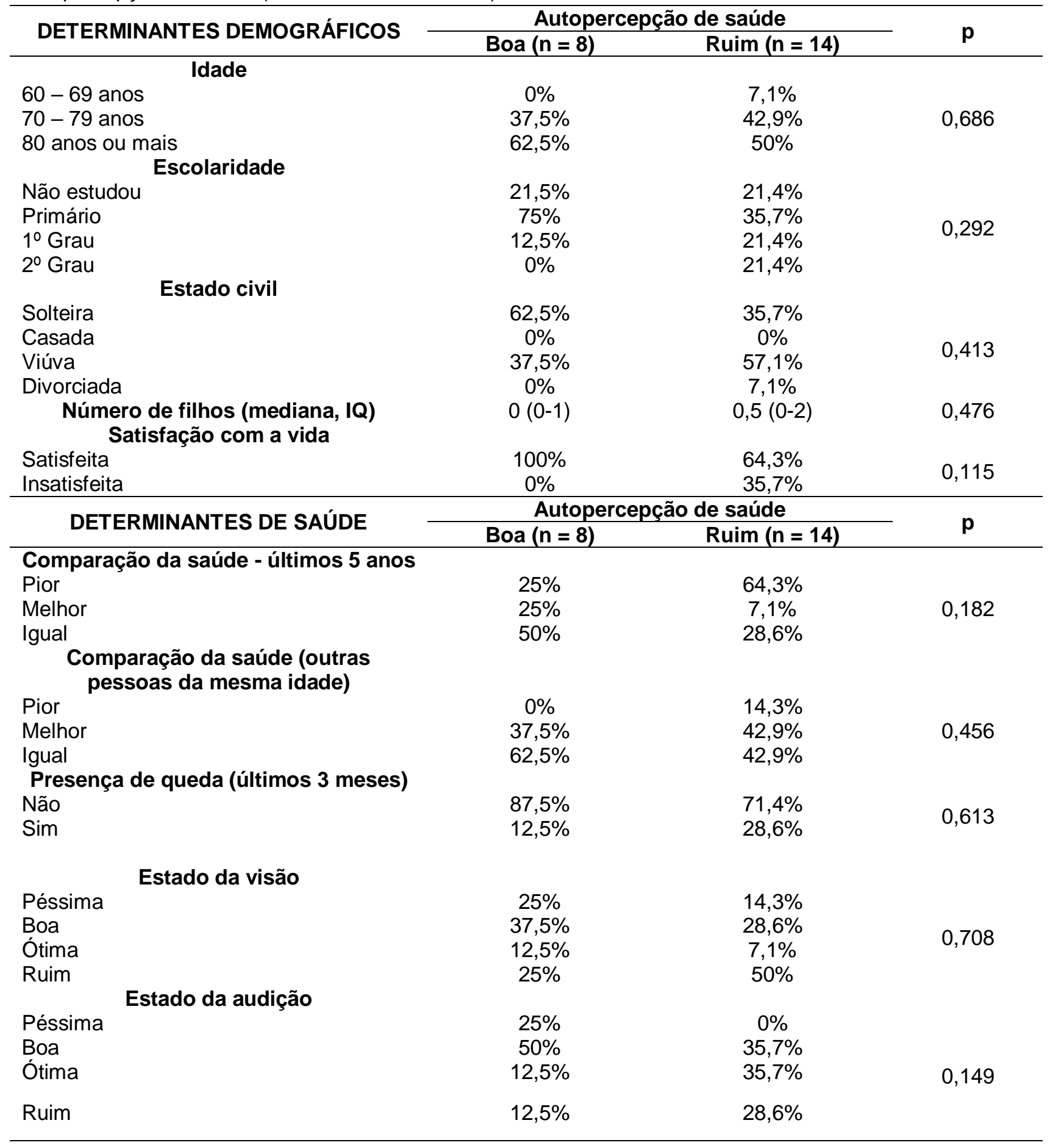




\begin{tabular}{|c|c|c|c|}
\hline Estado dos dentes & & & \multirow{5}{*}{0,545} \\
\hline Péssimo & $25 \%$ & $7,1 \%$ & \\
\hline Bom & $37,5 \%$ & $35,7 \%$ & \\
\hline Ótimo & $12,5 \%$ & $7,1 \%$ & \\
\hline Ruim & $25 \%$ & $50 \%$ & \\
\hline \multicolumn{4}{|l|}{ Episódios de perda de urina } \\
\hline Não & $62,5 \%$ & $35,7 \%$ & \multirow{2}{*}{0,378} \\
\hline Sim & $37,5 \%$ & $64,3 \%$ & \\
\hline \multirow{2}{*}{ DETERMINANTES SOCIAIS } & \multicolumn{2}{|c|}{ Autopercepção de saúde } & \multirow[b]{2}{*}{$\mathbf{p}$} \\
\hline & Boa $(n=8)$ & Ruim (n = 14) & \\
\hline \multicolumn{4}{|l|}{$\begin{array}{c}\text { Relacionamento com as pessoas que } \\
\text { mora }\end{array}$} \\
\hline Insatisfeita & $0 \%$ & $7,1 \%$ & \multirow{2}{*}{1,00} \\
\hline Satisfeita & $100 \%$ & $92,9 \%$ & \\
\hline \multicolumn{4}{|l|}{ Assistência familiar } \\
\hline Não & $75 \%$ & $42,9 \%$ & \multirow{2}{*}{0,204} \\
\hline & $25 \%$ & $57,1 \%$ & \\
\hline \multicolumn{4}{|l|}{ Visitas na última semana } \\
\hline Não & $50 \%$ & $7,1 \%$ & \multirow{2}{*}{0,039} \\
\hline Sim & $50 \%$ & $92,9 \%$ & \\
\hline \multirow{2}{*}{ DETERMINANTES ECONÔMICOS } & \multicolumn{2}{|c|}{ Autopercepção de saúde } & \multirow[b]{2}{*}{$\mathbf{p}$} \\
\hline & Boa $(n=8)$ & Ruim (n = 14) & \\
\hline \multicolumn{4}{|l|}{ Trabalho anterior } \\
\hline Não & $0 \%$ & $14,3 \%$ & \multirow{3}{*}{0,515} \\
\hline Sim & $100 \%$ & $85,7 \%$ & \\
\hline \multicolumn{3}{|l|}{ Ocupação atual } & \\
\hline Não & $100 \%$ & $100 \%$ & \multirow[b]{2}{*}{ - } \\
\hline Sim & $0 \%$ & $0 \%$ & \\
\hline \multicolumn{4}{|l|}{ Forma de sustento } \\
\hline Aposentadoria & $87,5 \%$ & $35,7 \%$ & \multirow{3}{*}{0,051} \\
\hline Pensão & $12,5 \%$ & $28,6 \%$ & \\
\hline $\begin{array}{l}\text { Aposentadoria + ajuda } \\
\quad \text { Renda mensal }\end{array}$ & $0 \%$ & $35,7 \%$ & \\
\hline Não sabe/Não respondeu & $50 \%$ & $28,6 \%$ & \multirow{3}{*}{0,485} \\
\hline Menor ou igual a 1 Salário Mínimo & $25 \%$ & $50 \%$ & \\
\hline \multirow{2}{*}{\multicolumn{4}{|c|}{$\begin{array}{c}\text { Comparação com situação econômica } \\
\text { de } 50 \text { anos }\end{array}$}} \\
\hline & & & \\
\hline Igual & $62,5 \%$ & $57,1 \%$ & \multirow{3}{*}{0,329} \\
\hline Melhor & $37,5 \%$ & $21,4 \%$ & \\
\hline \multirow{2}{*}{\multicolumn{4}{|c|}{$\begin{array}{c}\text { Renda em comparação com suas } \\
\text { necessidades }\end{array}$}} \\
\hline & & & \\
\hline Dá e sobra & $50 \%$ & $7,1 \%$ & \multirow{3}{*}{0,056} \\
\hline Dá na conta certa & $37,5 \%$ & $50 \%$ & \\
\hline Sempre falta & $12,5 \%$ & $42,9 \%$ & \\
\hline
\end{tabular}

Nota: $p=$ significância estatística.

Fonte: Dados dos Autores, 2018.

\section{CONSIDERAÇÕES FINAIS}

O estudo indica que idosas com maior interfêrencia nas atividades desempenhadas no dia a dia e na sociedade por dificuldades, incapacidades, transtornos ou dores causadas pelas doenças durante um período de tempo maior, como é o caso das doenças crônicas e perda funcional, tendem a autoperceber a saúde negativamente. Ficam, portanto, descritas as características da autopercepção de saúde observadas na população idosa institucionalizada estudada, para que futuras intervenções e condutas bem como 
planejamentos de assistência a esta população levem em consideração os principais pontos levantados e futuros estudos com populações maiores possam investigar as demais cacterísticas e necessidades dos idosos institucionalizados. Entre as limitações encontradas durante a realização desta pesquisa destaca-se a baixa adesão, o que repercutiu no tamanho da amostra, uma vez que a população inicial de 60 idosas teve o número reduzido a menos da metade de participantes que se encaixavam em todos os critérios de elegibilidade.

\section{REFERÊNCIAS}

1. Agência Nacional de Vigilância Sanitária. Resolução da Diretoria Colegiada № 283, de 26 de setembro de 2005. Regulamento técnico para o funcionamento das instituições de longa permanência para idosos. Brasília: ANVISA; 2005.

2. ALVES LC, et al. Fatores associados à incapacidade funcional dos idosos no Brasil: análise multinível. Rev. Saúde Pública, 2010; 44(3): 468-478.

3. ALVES LC, RODRIGUES RN. Determinantes da autopercepção de saúde entre idosos do Município de São Paulo, Brasil. Rev. Panam Salud Publica, 2005; 17(5-6): 333-341.

4. ARNADOTTIR SA, et al. Determinants of self-rated health in old age: a population-based, cross-sectional study using the International Classification of Functioning. BMC Public Health, 2011; 11: 670.

5. BELÉM PLO, et al. Autoavaliação do estado de saúde e fatores associados em idosos cadastrados na Estratégia Saúde da Família de Campina Grande, Paraíba. Rev. Bras. Geriatr Gerontol, 2016; 19(2): 265-276.

6. BERTOLUCCI PHF, et al. O Mini exame do estado mental em uma população geral. Arq. Neuropsiquiatr, 1994; 51(1): 1-7.

7. BESSA MEP, SILVA MJ. Motivações para o ingresso dos idosos em instituições de longa permanência e processos adaptativos: um estudo de caso. Texto contexto - Enferm, 2008; 7(2): 258-265.

8. BORGES AM, et al. Autopercepção de saúde em idosos residentes em um município do interior do Rio Grande do Sul. Rev Bras Geriatr Gerontol, 2014; 17(1): 79-86.

9. BORGES JBC. Avaliação da medida de independência funcional - escala MIF- e qualidade de serviço - escala servqual - em cirurgia cardíaca. Tese (Doutorado em Bases Gerais de Cirurgia) - Faculdade de Medicina de Botucatu da Universidade Estadual Paulista, Botucatu, 2006; 118 p.

10. BRASIL. Diretrizes e normas regulamentadoras de pesquisas envolvendo seres humanos. Resolução $n=466$, de 12 de dezembro de 2012. Brasília: Conselho Nacional de Pesquisa, 2012. Disponível em: http://bvsms.saude.gov.br/bvs/saudelegis/cns/2013/res0466_12_12_2012.html. Acesso em: 15 nov. 2018.

11. DAMIÁN J, et al. Factors associated with self-rated health in older people living in institutions. BMC Geriatr, 2008; 8(5): $1-6$.

12. DANTAS CMHL, et al. Capacidade funcional de idosos com doenças crônicas residentes em Instituições de Longa Permanência. Rev Bras Enferm, 2013; 66(6): 914-20.

13. DIAS DSG, et al. Comparação da percepção subjetiva de qualidade de vida e bem-estar de idosos que vivem sozinhos, com a família e institucionalizados. Rev Bras Geriatr Gerontol, 2013; 16(1): 127-138.

14. Instituto Brasileiro de Geografia e Estatística. Projeção da população por sexo e grupos de idade, em 1ำ de julho 2000-2060. Disponível em: http://www.ibge.gov.br/home/estatistica/populacao/projecao_da_ população/2013/default_tab.shtm. Acesso em: 15 dez. 2018.

15. Instituto Brasileiro de Geografia e Estatística. Pesquisa Nacional de Saúde 2013: percepção do estado de saúde, estilos de vida e doenças crônicas - Brasil, Grandes Regiões e Unidades da Federação. Rio de Janeiro: Instituto Brasileiro de Geografia e Estatística; 2014. 181 p. Disponível em: ftp://ftp.ibge.gov.br/PNS/2013/ pns2013.pdf. Acesso em: 23 nov. 2018.

16. LIMA CLJ, et al. Perfil sociodemográfico e clínico de idosos institucionalizados. Rev Enferm UFPE, 2013; 7(10): 6027-6034.

17. LIMA-COSTA MF, et al. A estrutura da autoavaliação da saúde entre idosos: projeto Bambuí. Rev Saúde Pública, $2004 ; 38(6): 827-834$.

18. NUNES APN, et al. Relações sociais e autopercepção da saúde: projeto envelhecimento e saúde. Rev Bras Epidemiol, 2012; 15(2): 415-428.

19. VERAS R. Envelhecimento populacional contemporâneo: demandas, desafios e inovações. Rev Saúde Públic, 2009; 43(3): 548-554.

20. WEISS SLI. Categorização do Questionário Brazil Old Age Schedule (BOAS) para estudos na área do desenvolvimento humano. EFDeportes Revista Digital, 2013; 17(177). 